\title{
Moyamoya Disease Presenting as Transient Amnesia with Cortical Blindness
}

\author{
Jun Yeong Hong ${ }^{*}$ (D), Sang-Jun Na (D), Jung-Won Shin ${ }^{\dagger}$ (D), Hyun-Sook $\operatorname{Kim}^{\dagger}$ (D), Won Chan $\operatorname{Kim}^{\dagger}$ (D), \\ Ok-Joon Kim ${ }^{\dagger}$ (D), Seung-Hun $\mathrm{Oh}^{\dagger}$ (D), Kee Ook Lee ${ }^{\dagger}$ (D) \\ Department of Neurology, Konyang University College of Medicine*, Daejeon; Department of Neurology, CHA Bundang Medical Center, \\ $\mathrm{CHA}$ University ${ }^{\dagger}$, Seongnam, Korea
}

Symptoms of moyamoya disease (MMD) are typically associated with regions of the brain supplied by the internal carotid arteries and middle cerebral arteries. We describe a patient who presented with transient cortical blindness, anosognosia, and amnesia associated with MMD. A 22-year-old right-handed woman presented with amnesia and visual impairments. She completely denied blindness and recent memory disturbances with confabulation. Diffusion and T2-weighted magnetic resonance imaging showed high signal intensities on the left basal ganglia, suggesting an acute ischemic stroke. In cerebral angiography, complete occlusion of the distal internal carotid artery was shown, with collateral flow noted within the posterior cerebral artery. Neuropsychological and cognitive problems related to MMD have been previously reported in the literature. We believe that these symptoms reflect transient ischemia caused by a disturbed hemodynamic status. This case report demonstrates that patients with MMD can present with transient posterior circulation symptoms in the form of Dide-Botcazo syndrome.

J Neurosonol Neuroimag 2019;11(2):169-172

Key Words: Moyamoya disease; Blindness, Cortical; Amnesia; Cerebral infarction
Received: October 29, 2019

Revised: December 4, 2019

Accepted: December 4, 2019

Address for correspondence: Kee Ook Lee

Department of Neurology, CHA Bundang Medical Center, CHA University, 59 Yatap-ro, Bundang-gu, Seongnam 13496, Korea

Tel: $+82-31-780-1975$,

Fax: $+82-31-780-5269$

E-mail: niceiatros@cha.ac.kr
Moyamoya disease (MMD) is a rare progressive occlusive cerebrovascular disease characterized by occlusion or stenosis of the internal carotid artery and the compensatory development of abnormal Moyamoya vessels at the base of brain, originating either from the basilar artery or extracranial blood vessels. ${ }^{1}$ The annual prevalence and incidence of MMD are estimated to be 3.16 and 0.35 per 100,000 individuals, respectively.,3 Symptoms and signs of MMD are presented as variable etiological categories. Symptoms of MMD are typically associated with the regions of the brain supplied by the internal carotid arteries and middle cerebral arteries. ${ }^{2}$

Patients with bilateral occipital and temporal lesions typically present with acute onset of cortical blindness, anosognosia, and amnesia. We now describe a patient with MMD and unusual symptoms who presented with transient cortical blindness, anosognosia, and amnesia after experiencing emotional stress.

\section{CASE REPORT}

A 22-year-old right-handed woman was admitted to the emergency department 4 hours after the sudden onset of amnesia and visual impairment. There was no history of head trauma or and no remarkable previous medical or family history. She completely denied disturbances of recent memory with confabulation. However, her friend said that she showed visual disturbances, abnormal behaviors, and amnesia which preceded an episode of emotional stress. Before the symptoms developed, she was angry and crying hysterically, and her friend reported comforting her. Upon presentation to the emergency room (ER) she exhibited anxiety and hyperventilation syndrome. Later, she developed visual disturbances accompanied by amnesia and abnormal behaviors. She continually repeated the same questions and had difficulty forming new memories. The patient 
could not recall anything that happened after the onset of her symptoms. She was unable to recall three words 5 minutes after demonstrating successful registration. She exhibited severe deficits in naming and object use, suggesting visual agnosia. She continued to deny visual loss, which was initially believed to be due to the patient's confusion. Both direct and indirect pupil reactions were healthy, and no abnormal eye movements were apparent. Examinations of the patient's cranial nerves and limbs were unremarkable. An electrocardiography showed normal sinus rhythm. Laboratory tests and cerebrospinal fluid analysis for inflammatory-autoimmune diseases were also unremarkable.

Magnetic resonance imaging (MRI) was performed 3 hours after arrival at the ER. Diffusion-weighted and T2-weighted MRI images showed a high signal intensity lesion on left basal ganglia which suggested an acute cerebral infarction. A T2-weighted MRI image also demonstrated a high signal intensity lesion in the right parieto-occipital region, suggesting an ischemic origin (Fig. 1). Magnetic resonance and conventional angiog- raphy showed a severely stenotic and occlusive lesion and rete formation in the bilateral middle cerebral and internal carotid arteries, which suggested MMD (Fig. 2). Perfusion weighted image (PWI) demonstrated hypoperfusion in the posterior cerebral and middle cerebral vascular territories (Fig. 1). The patient's brain electroencephalogram and transthoracic echocardiogram were all within normal limits.

The next day, her blindness and amnesia were resolved. A neuropsychological test revealed a normal score on day 4. She performed very well in all cognitive tasks, including language, global efficiency, gestural praxis, and verbal and visual memory. However, she experienced three attacks of transient weakness in her limbs during the 2-year follow-up period after the first symptom onset. Ultimately, prophylactic bilateral extracranial-intracranial artery bypass surgery was performed, and the transient ischemic symptoms completely disappeared after surgery.
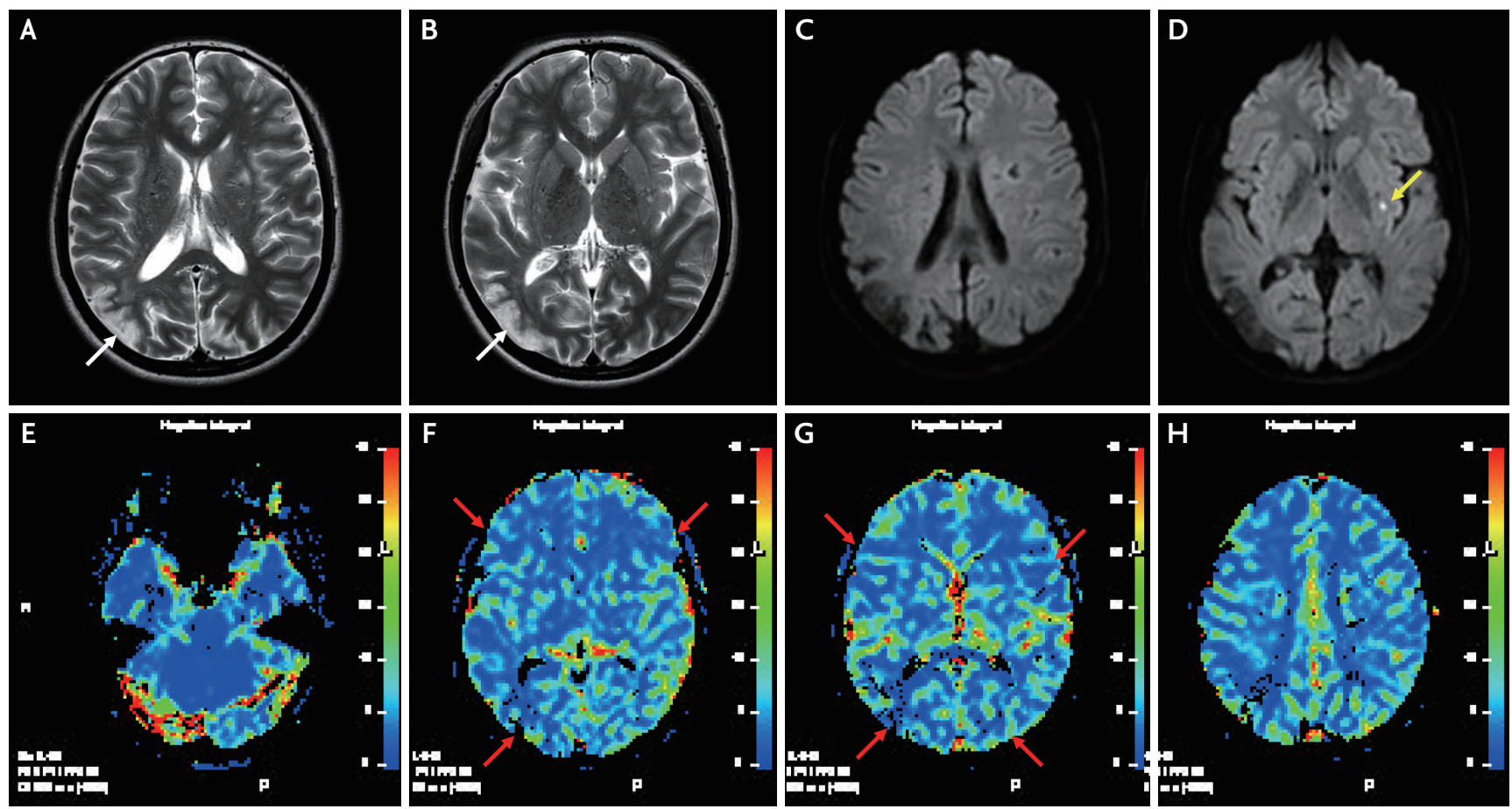

FIG. 1. MRI of the brain. (A, B) Axial T2 and (C, D) diffusion-weighted MRI show acute cerebral infarction on the left basal ganglia (yellow arrow of D). T2-weighted MRI image also shows a high signal intensity lesion in the right parieto-occipital regions (white arrow of A, B). (E-H) Perfusion weighted image demonstrates reduced CBF in the bilateral posterior cerebral and middle cerebral vascular territories (red arrows of F, G). MRI; magnetic resonance imaging, CBF; cerebral blood flow. 


\section{DISCUSSION}

The neuropsychological or cognitive problems related to MMD were previously reported in the literature. ${ }^{4,5}$ Patients with MMD are more likely to have neuro-psychological sequelae. In general, cognitive impairments associated with MMD have been described as neuro-psychologic sequelae that occur after stroke. These can manifest as disturbances of memory, attention, performance, and social behavior in pediatric cases. ${ }^{5}$ However, recently some adult cases have been reported and suggest that neurocognitive disorders can occur without radiological evidence of major strokes. ${ }^{4}$ The association between brain dysfunction and persistent frontal lobe hemodynamic compromise can be demonstrated using single-photon emission computed tomography, even if no cerebral infarction has occurred. ${ }^{4}$

However, to our knowledge, these presented problems, which mimic Dide-Botcazo syndrome, may not have been reported in previous literature. Dide-Botcazo syndrome is a rare clinical syndrome characterized by a combination of cortical blindness with anosognosia for blindness, retro-anterograde amnesia, topographic agnosia, alexia, and astereognosis secondary to bilateral occipital cortex lesions which also involve the inferior-medial temporal lobe structure. ${ }^{6,7}$ These symptoms of Dide-Botcazo syndrome are probably due to transient ischemia caused by disturbed hemodynamic status.

The clinical signs of MMD are presumably caused by the chronic hemodynamic compromise known to occur in steno-occlusive cerebrovascular disease. MMD lesions are located primarily in the frontal and parietal watershed regions and may affect the deep or superficial gray matter. ${ }^{8}$ We suggested that the posterior circulation symptoms or visual impairments also could be a major initial manifestation of the MMD. Moreover, acute onset of cortical blindness, anosognosia, and amnesia are commonly encountered in patients with bilateral occipital and temporal lesions. When the middle cerebral artery is occluded, hemodynamic stress on the anastomotic vessel may be increased. Impaired cerebrovascular reactivity tends to spontaneously improve with time and with the formation of collateral circulation. In general, when the MMD progresses, the posterior cerebral artery (PCA) develops as collateral flow and supplies blood to affected brain regions; however, in this case, the PCA flow was not abundantly developed.

For this reason, the patient may have developed hyperventilation-induced hypo-perfusion symptoms related to posterior circulation. Moreover, transient symptoms may occur due to temporary deterioration of posterior circulation in the presence of increased hemodynamic stress. Only symptoms related to posterior circulation developed in this patient, even though perfusion of the whole brain was reduced in PWI. A possible explanation for this phenomenon is that the posterior circulation under hemodynamic stress may be less tolerated than that of the anterior circulation. However, this was an initial transient symptom, and the patient later developed three attacks of transient weakness that might be considered symptoms related to anterior cir-
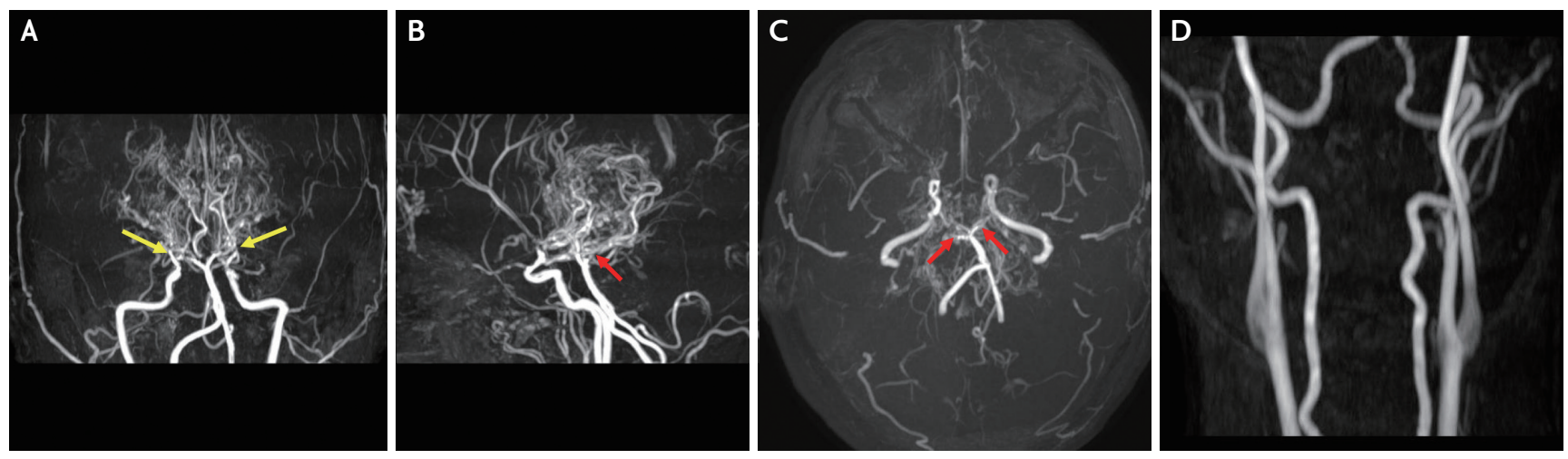

FIG. 2. MRA of head and neck. (A-D) MRA reveals severe stenotic and occlusive lesion and rete formation in bilateral MCA and ICA (yellow arrows of A). MRA also shows bilateral PCA steno-occlusive lesions with minimal leptomeningeal collaterals (red arrows of $B, C)$. MRA; magnetic resonance angiography, MCA; middle cerebral artery, ICA; internal carotid artery, PCA; posterior cerebral artery. 
culation. Further research with a larger set of clinical data and more individual PWI analyses is required to confirm the effects of hemodynamic stress on anterior and posterior circulation in patients with MMD.

In the case of neuropsychological and cognitive abnormalities after emotional stress, not only the psychologic problem or the hyperventilation syndrome should be considered but also the various causes, including stroke.

\section{Conflicts of Interest}

No potential conflicts of interest relevant to this article was reported.

\section{REFERENCES}

1. Suzuki J, Takaku A. Cerebrovascular "Moyamoya" disease. Disease showing abnormal net-like vessels in base of brain. Arch Neurol. 1969;20:288-299.

2. Kim JS. Moyamoya disease: Epidemiology, clinical features, and diagnosis. J Stroke. 2016;18:2-11.

3. Wakai K, Tamakoshi A, Ikezaki K, Fukui M, Kawamura T, Aoki R, et al. Epidemiological features of Moyamoya disease in Japan: findings from a nationwide survey. Clin Neurol Neurosurg. 1997;99 Suppl 2:S1-S5.

4. Nakagawara J, Osato T, Kamiyama K, Honjo K, Sugio H, Fumoto K, et al. Diagnostic imaging of higher brain dysfunction in patients with adult Moyamoya disease using statistical imaging analysis for [123i]iomazenil single photon emission computed tomography. Neurol Med Chir (Tokyo). 2012;52:318-326.

5. Matsushima Y, Aoyagi M, Masaoka H, Suzuki R, Ohno K. Mental outcome following encephaloduroarteriosynangiosis in children with Moyamoya disease with the onset earlier than 5 years of age. Childs Nerv Syst. 1990;6:440-443.

6. Lazzarino De Lorenzo LG, Ffytche DH, Di Camillo E, Buiatti T. The dide-botcazo syndrome: forgotten and misunderstood. Cortex. 2014;56:182-190.

7. Cappellari M, Tomelleri G, Di Matteo A, Carletti M, Magalini A, Bovi P, et al. Dide-botcazo syndrome due to bilateral occlusion of posterior cerebral artery. Neurol Sci. 2010;31:99101.

8. Jin Q, Noguchi T, Irie H, Kawashima M, Nishihara M, Takase Y, et al. Assessment of Moyamoya disease with 3.0-t magnetic resonance angiography and magnetic resonance imaging versus conventional angiography. Neurol Med Chir (Tokyo). 2011;51:195-200. 